\title{
Assessing the Potential of Network Reconfiguration to Improve Distributed Generation Hosting Capacity in Active Distribution Systems
}

\author{
Florin Capitanescu, Luis F. Ochoa, Senior Member, IEEE, Harag Margossian, and \\ Nikos D. Hatziargyriou, Fellow, IEEE
}

\begin{abstract}
As the amount of distributed generation (DG) is growing worldwide the need to increase the hosting capacity of distribution systems without reinforcements is becoming nowadays a major concern. This paper explores how the DG hosting capacity of active distribution systems can be increased by means of network reconfiguration, both static, i.e., grid reconfiguration at planning stage, and dynamic, i.e., grid reconfiguration using remotely controlled switches as an active network management (ANM) scheme. The problem is formulated as a mixed-integer, nonlinear, multi-period optimal power flow (MP-OPF) which aims to maximize the DG hosting capacity under thermal and voltage constraints. This work further proposes an algorithm to break-down the large problem size when many periods have to be considered. The effectiveness of the approach and the significant benefits obtained by static and dynamic reconfiguration options in terms of DG hosting capacity are demonstrated using a modified benchmark distribution system.
\end{abstract}

Index Terms-active distribution system, distributed generation, hosting capacity, network switching, optimal power flow, smart grids.

\section{NOMENCLATURE}

List of Acronyms

$\begin{array}{ll}\text { ANM } & \text { active network management. } \\ \text { APFC } & \text { adaptive power factor control. } \\ \text { DG } & \text { distributed generation. } \\ \text { DSO } & \text { distribution system operator. } \\ \text { MHC } & \text { maximum hosting capacity. } \\ \text { MINLP } & \text { mixed integer nonlinear programming. } \\ \text { NLP } & \text { nonlinear programming. } \\ \text { OLTC } & \text { on load tap changing transformer. } \\ \text { OPF } & \text { optimal power flow. } \\ \text { MP-OPF } & \text { multi-period optimal power flow. } \\ \text { RCS } & \text { remotely controlled switches. }\end{array}$

Sets

$N \quad$ set of nodes.

$G \quad$ set of DG units.

F. Capitanescu is an independent researcher (e-mail: fcapitanescu@yahoo.com). L. Ochoa is with the University of Manchester, UK (e-mail: luis_ochoa@ieee.org). H. Margossian is with both SnT, University of Luxembourg, Luxembourg, and K.U. Leuven, Belgium, (e-mail: harag.margossian@uni.lu). N. Hatziargyriou is with NTUA, Greece (e-mail: nh@power.ece.ntua.gr). F. Capitanescu and H. Margossian are partly supported by the Fonds National de la Recherche, Luxembourg, as a part of the "Reliable and Efficient Distributed Electricity Generation in Smart Grids" (REDESG) project (C11/SR/1278568).
$E$ set of substations interconnecting the distribution network with the upstream network.

$T$ set of OLTC transformers.

$L \quad$ set of lines.

$S \quad$ subset of lines with remotely controlled switches.

$M \quad$ set of periods.

\section{Continuous optimization variables}

$P_{g i} \quad$ installed active power capacity of DG unit at a predefined location $i$.

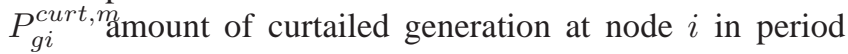
$m$.

$P_{e i}^{m} \quad$ active power of the substations interconnecting with the upstream grid in period $m$.

$Q_{e i}^{m} \quad$ reactive power of the substations interconnecting with the upstream grid in period $m$.

$\phi_{g i}^{m} \quad$ phase angle between voltage and current in period $m$ which defines DG power factor $\cos \left(\phi_{g i}^{m}\right)$.

$e_{i}^{m} \quad$ the real component of complex voltage at bus $i$ in period $m$.

$f_{i}^{m} \quad$ the imaginary component of complex voltage at bus $i$ in period $m$.

\section{Binary optimization variables}

$s_{i j} \quad$ binary variable that models the on/off status of a line switch over all periods (in static reconfiguration).

$s_{i j}^{m} \quad$ binary variable that models the on/off status of a line switch in period $m$ (in dynamic reconfiguration).

\section{Parameters}

$g_{i j} \quad$ conductance of the branch linking nodes $i$ and $j$.

$b_{i j} \quad$ susceptance of the branch linking nodes $i$ and $j$.

$b_{i j}^{s h} \quad$ shunt susceptance of the branch linking nodes $i$ and $j$.

$P_{c i} \quad$ peak load active power.

$Q_{c i} \quad$ peak load reactive power.

$\omega_{g i}^{m} \quad$ scalar $\left(\omega_{g i}^{m} \in[0 ; 1]\right)$, allowing to define the generation level in period $m$ relative to the installed power, as $\omega_{g i}^{m} P_{g i}$ and $\omega_{g i}^{m} P_{g i} \tan \left(\phi_{g i}^{m}\right)$, respectively.

$\eta_{m} \quad$ scalar $\left(\eta_{m} \in[0 ; 1]\right)$, allowing to define the load level in period $m$ relative to the peak, as $\eta_{m} P_{c i}$ and $\eta_{m} Q_{c i}$, respectively.

$V_{i \text { min }}$ minimum voltage limit. 
$V_{i \max }$ maximum voltage limit.

$I_{i j}^{\max }$ maximum current of line linking nodes $i$ and $j$.

$K_{I} \quad$ "bigM"-type constant.

$\phi_{g i}^{\min }$ minimum phase angle between voltage and current.

$\phi_{g i}^{\max }$ maximum phase angle between voltage and current.

$\tau^{m} \quad$ duration of the period $m$.

$\gamma \quad$ scalar $(\gamma \in[0 ; 1])$ defining the maximum allowed amount of curtailed energy relative to the unconstrained energy harvest over all periods.

$\tilde{s}_{i j} \quad$ switches status in DSO "business as usual" topology.

$\Delta s^{s t a}$ maximum allowed number of topology changes in static reconfiguration.

$\Delta s^{d y n}$ maximum allowed number of topology changes in dynamic reconfiguration.

\section{INTRODUCTION}

$\mathbf{T}$ HE increase of the amount of distributed generation (DG) (e.g., wind, photovoltaic, etc.) at both medium and low voltages is encouraged worldwide as a means to meet today's stringent environmental constraints. DG installations are mainly limited by operational constraints, like thermal limits, voltage rise, or protection issues. The adoption of the "fit-and-forget" principle, i.e., allowing accommodation of DG only if it does not lead to constraint violation under the worst-case scenario, leads, in many cases, to costly network reinforcements or limited DG capacity allowed to be connected. Furthermore, where unbundling rules apply (as in Europe), distribution system operators (DSOs) have generally little or no control over DG placement and size, provided that grid operational constraints are satisfied. In this context, non-optimal DG locations may have negative consequences in several respects: (i) failure to achieve green energy targets, especially in countries with modest renewable energy resources, and (ii) limited harvesting of the potential DG benefits (e.g., deferral of load-led network reinforcements, reduction in losses and carbon emissions, etc.).

To overcome the drawbacks of the "fit-and-forget" approach, active network management (ANM) schemes have been proposed as a solution to significantly increase the amount of DG capacity, while exploiting DG benefits. Within this new paradigm, centralized [1] or distributed [2] control schemes have been proposed, together with additional communication, monitoring and control infrastructure, so as to manage DG output and other potentially controllable network elements (e.g., on-load tap changing transformers, etc.).

Although DG location and size is decided by the DG owner according to the site climatic conditions, gas supplies and other techno-economic criteria, differentiated connection charges and regulatory rules can influence DG location decision. Therefore, appropriate tools to determine the most suitable locations and corresponding penetrations are very useful [3]-[5]. The assessment of the distribution system DG hosting capacity and the closely related problem of optimal DG siting and sizing have become a major research focus [3][5]. Several approaches have been investigated in this context including: linearized OPF [6], [7], snapshot-based NLP OPF with additional constraints (e.g., on fault level [8]), multiperiod NLP OPF [9], [10], multi-period MINLP OPF [11], and snapshot-based metaheuristics (e.g., genetic algorithm [12][14]).

However, these previous works did not explore available options to increase DG hosting capacity by network reconfiguration. This idea has been articulated recently [15], [16] and its potential benefits have been illustrated, on a snapshot-basis, by simple topology enumeration [15] or genetic algorithm [16].

Network reconfiguration is a major DSO control means which is used for various purposes such as: loss minimization [17], [18], load balancing [18], post-fault service restoration [19]-[21], reliability improvement [22], or multi-criteria analysis [22]. According to the distribution system operation time frame network reconfiguration can be classified as:

- static reconfiguration which considers all (manually or remotely controlled) switches and looks for an improved fixed topology at the planning stage (e.g., from an yearly/seasonal basis up to operational planning);

- dynamic reconfiguration which considers remotely controlled switching (RCS) in a centralized active network management (ANM) scheme to remove grid congestions in real time.

Static network reconfiguration has been very extensively investigated so far, for snapshot-based loss minimization, relying on approaches such as: mathematical programming (e.g., MINLP with complex voltages expressed in polar coordinates [23], Benders decomposition approach applied to MINLP formulation [24], mixed-integer conic programming [25], [26], mixed-integer linear programming [25], [27], mixed-integer quadratically constrained programming [28], etc.) or heuristic techniques (e.g., branch exchange [18], [22], [29], [30], genetic algorithm [21], [31], memetic algorithm [21], informed search [20], harmony search algorithm [17], etc.).

Dynamic network reconfiguration has comparatively received much less attention and has been studied using approaches such as: linear programming [32], dynamic programming [19], [33], or branch exchange-based heuristics [22], [29].

The work described in this paper builds upon the NLP multiperiod OPF framework proposed in [9] by which centralized ANM schemes are considered to cope with voltage rise and thermal overload issues. The main contribution of this work is that it further investigates, by means of a MINLP MP-OPF formulation, the extent of the potential benefits from adopting static and dynamic network reconfiguration as options to increase the ability of distribution systems to host DG. Another contribution of this work is that it proposes an algorithm for reducing the size of the MP-OPF for a large number of periods.

It is important to emphasize that, compared to most related works in network reconfiguration, the proposed approach considers: a different optimization goal, distributed generation, the inherent time-varying (renewable) generation patterns and load behavior which are aggregated into multi time periods, ANM schemes, and both static and dynamic reconfiguration options. Given these salient features and the adoption of rectangular coordinates for voltages, the proposed problem formulation differs fundamentally from other MINLP-based works on network reconfiguration such as [23], [24]. 


\section{Accepted Paper}

The remaining of this work is organized as follows. Section II presents the mathematical model of the MP-OPF problem. Section III describes an algorithm to reduce the problem size. Section IV provides extensive numerical results with the method from the application of the approach on the widely used 34-bus system from [18]. Section V discusses different implementation aspects of the method. Finally, conclusions are drawn in Section VI.

\section{PROBLEM FORMULATION}

The optimization problem is formulated relying on rectangular coordinates of complex voltages to alleviate problem nonlinearity:

$$
\underline{V}_{i}=e_{i}+j f_{i}, \quad V_{i}=\sqrt{e_{i}^{2}+f_{i}^{2}}, \quad i \in N
$$

where $e_{i}$ and $f_{i}$ are the real and imaginary components respectively, and $V_{i}$ is the voltage magnitude.

\section{A. Objective function}

The goal of the optimization problem is to maximize the overall amount of DG that can be hosted by the distribution system. The corresponding objective function is called hereafter maximum hosting capacity (MHC):

$$
M H C=\max \sum_{i \in G} P_{g i}
$$

where $P_{g i}$ denotes the installed active power capacity of DG unit at a predefined location $i$.

Although this formulation can account for discrete $\mathrm{DG}$ capacities [11], these are modelled as continuous variables. This is due to the fact that this formulation aims to model the hosting capabilities of the network rather than actually allocating DG plants. In addition, this also reduces the computational burden of the problem.

\section{B. Constraints}

1) Power flow equations: the active/reactive power balance equations at bus $i \in N$ in each period $m \in M$ are:

$$
\begin{aligned}
& P_{e i}^{m}+\omega_{g i}^{m} P_{g i}-P_{g i}^{c u r t, m}-\eta_{m} P_{c i}= \\
& \sum_{j \in N} s_{i j}^{m} P_{i j}^{m}=\sum_{j \in N} s_{i j}^{m} g_{i j}\left(V_{i}^{m}\right)^{2} \\
& -\sum_{j \in N} s_{i j}^{m}\left[\left(e_{i}^{m} e_{j}^{m}+f_{i}^{m} f_{j}^{m}\right) g_{i j}+\left(f_{i}^{m} e_{j}^{m}-e_{i}^{m} f_{j}^{m}\right) b_{i j}\right], \\
& Q_{e i}^{m}+\omega_{g i}^{m} P_{g i} \tan \left(\phi_{g i}^{m}\right)-\eta_{m} Q_{c i}= \\
& \sum_{j \in N} s_{i j}^{m} Q_{i j}^{m}=-\sum_{j \in N} s_{i j}^{m}\left(b_{i j}^{s h}+b_{i j}\right)\left(V_{i}^{m}\right)^{2} \\
& +\sum_{j \in N} s_{i j}^{m}\left[\left(e_{i}^{m} e_{j}^{m}+f_{i}^{m} f_{j}^{m}\right) b_{i j}-\left(f_{i}^{m} e_{j}^{m}-e_{i}^{m} f_{j}^{m}\right) g_{i j}\right],
\end{aligned}
$$

where, $P_{i j}^{m}$ and $Q_{i j}^{m}$ denote the active and reactive power flows between nodes $i$ and $j$. Although the optimization model adopts a constant power load model, other load models (e.g., voltage dependent load model) can be incorporated straightforwardly.
2) Active/reactive powers on the substations interconnecting with the upstream grid:

$$
\begin{aligned}
& P_{e i}^{\min } \leq P_{e i}^{m} \leq P_{e i}^{\max }, i \in E, m \in M \\
& Q_{e i}^{\min } \leq Q_{e i}^{m} \leq Q_{e i}^{\max }, i \in E, m \in M
\end{aligned}
$$

3) Branch current limits:

$$
\begin{aligned}
& \left(I_{i j}^{m}\right)^{2}=\left(g_{i j}^{2}+b_{i j}^{2}\right)\left[\left(V_{i}^{m}\right)^{2}+\left(V_{j}^{m}\right)^{2}-2\left(e_{i}^{m} e_{j}^{m}+f_{i}^{m} f_{j}^{m}\right)\right] \\
& \leq K_{I}\left(1-s_{i j}^{m}\right)+s_{i j}^{m}\left(I_{i j}^{\max }\right)^{2}, i j \in L, m \in M,
\end{aligned}
$$

where $K_{I}$ is a "bigM"-type constant properly chosen to relax constraints (6), if line $i j$ is open at the optimal solution, a fact which is obtained as a result of the solution.

4) Voltage limits:

$$
V_{i \min }^{2} \leq\left(e_{i}^{m}\right)^{2}+\left(f_{i}^{m}\right)^{2} \leq V_{i \max }^{2}, i \in N, m \in M .
$$

5) Static reconfiguration:

$$
\sum_{i j \in L}\left|s_{i j}-\tilde{s}_{i j}\right| \leq \Delta s^{s t a},
$$

where $\tilde{s}_{i j}$ models the DSO "business as usual" topology and $s_{i j}$ models an improved fixed topology over all periods. This constraint expresses the fact that the DSO is not willing to perform static grid reconfiguration by using more than $\Delta s^{\text {sta }}$ actions on manually or remotely controlled switches.

6) Radiality: Because most distribution systems operate radially as a trade-off between investment cost (mainly in protection systems) and reliability, radiality is considered a constraint, which is modeled in the following way:

$$
\sum_{i j \in L} s_{i j}=\sum_{i j \in L} \tilde{s}_{i j} .
$$

The above constraint models the fact that the sum of statuses of all lines must not change after static reconfiguration. However, this constraint may be insufficient to ensure radiality in grids where there are some zero-injection nodes [28]. Actually, the presence of zero-injection nodes could lead to tricky cases because, at the optimal solution, these nodes can be isolated whereas the network remains in a weakly meshed configuration if this leads to a better objective and satisfies the problem constraints. A practical solution is adopted by which each zero-injection bus is replaced with a very small reactive power load (of value slightly above the power flow convergence tolerance), enforcing thereby that, in order to satisfy power flow equations, the node is never isolated. As the number of zero-injection nodes in a distribution system is generally small, this change does not affect practically the result of the optimization.

\section{Active network management}

To assess the MHC improvement in the presence of ANM schemes the corresponding constraints are modeled as follows.

1) Voltage control (VC): The control of the secondary voltage of the OLTC transformers can be incorporated into the model by choosing properly the bounds $V_{i \text { min }}$ and $V_{i \max }$ in constraints (7) for the corresponding busbar. 
2) Adaptive power factor control (APFC): Control of the DG power factor $\cos \left(\phi_{g i}^{m}\right)$ within some agreed range (e.g., between 0.95 lagging and 0.95 leading) can be modeled as:

$$
\phi_{g i}^{\min } \leq \phi_{g i}^{m} \leq \phi_{g i}^{\max }, i \in G, m \in M
$$

3) Energy curtailment (EC): Curtailment of DG power output can be limited to avoid economic unviability. This is modelled with the following constraint:

$$
\sum_{m \in M} P_{g i}^{c u r t, m} \tau^{m} \leq \gamma \sum_{m \in M} \omega_{g i}^{m} P_{g i} \tau^{m}, i \in G
$$

where $\tau^{m}$ is the duration of the period $m, \gamma$ is a scalar managing the amount of curtailed energy relative to the unconstrained energy harvest over all periods $\sum_{m \in M} \omega_{g i}^{m} P_{g i} \tau^{m}$.

Furthermore the active power curtailment of DG units is upper bounded by the DG plant production in period $m$ :

$$
P_{g i}^{\text {curt }, m} \leq \omega_{g i}^{m} P_{g i}, i \in G, m \in M .
$$

4) Dynamic reconfiguration: The ability of the DSO to actively operate RCS to remove constraints in real-time can be modeled by the following constraint:

$$
\sum_{i j \in S}\left|s_{i j}^{m}-s_{i j}^{m-1}\right| \leq \Delta s^{d y n}, i j \in S, m \in M
$$

This constraint models the DSO practical requirement to perform a limited number of switching actions $\Delta s^{d y n}$ to transfer from one state to another, where $s_{i j}^{m}$ models the possibility to act on RCS in period $m$.

Note that this constraint is different from the static reconfiguration (8), as it considers only RCS and is period-dependent.

Furthermore, this constraint imposes additional radiality constraint:

$$
\sum_{i j \in S} s_{i j}^{m}=\sum_{i j \in S} s_{i j}, m \in M,
$$

This models the fact that the sum of statuses of lines with RCS must not change after reconfiguration at every period $m$.

\section{Summary of control variables}

The set of control variables of this optimization problem comprises continuous variables $\left(P_{g i}, P_{g i}^{\text {curt }, m}, \phi_{g i}^{m}, P_{e i}^{m}, Q_{e i}^{m}\right.$, and $e_{i}^{m}, f_{i}^{m}$ at nodes where voltage is controlled) and binary variables $\left(s_{i j}^{m}\right.$ and $\left.s_{i j}\right)$, whereas $e_{i}^{m}$ and $f_{i}^{m}$ at all nodes are optimization variables.

\section{E. Overview of the proposed method}

An overview diagram of the proposed method in terms of data inputs and operational options is given in Fig. 1.

\section{REDUCING THE PROBLEM SIZE}

The MP-OPF formulation (1)-(14) is a very challenging MINLP problem due to the incorporation of network reconfiguration. Indeed, this, in combination with the number of periods to be considered, may lead to a very large combinatorial space. As a consequence, the size of the problem becomes significantly large and potentially unmanageable by current commercial solvers. An effective reduction of periods is hence

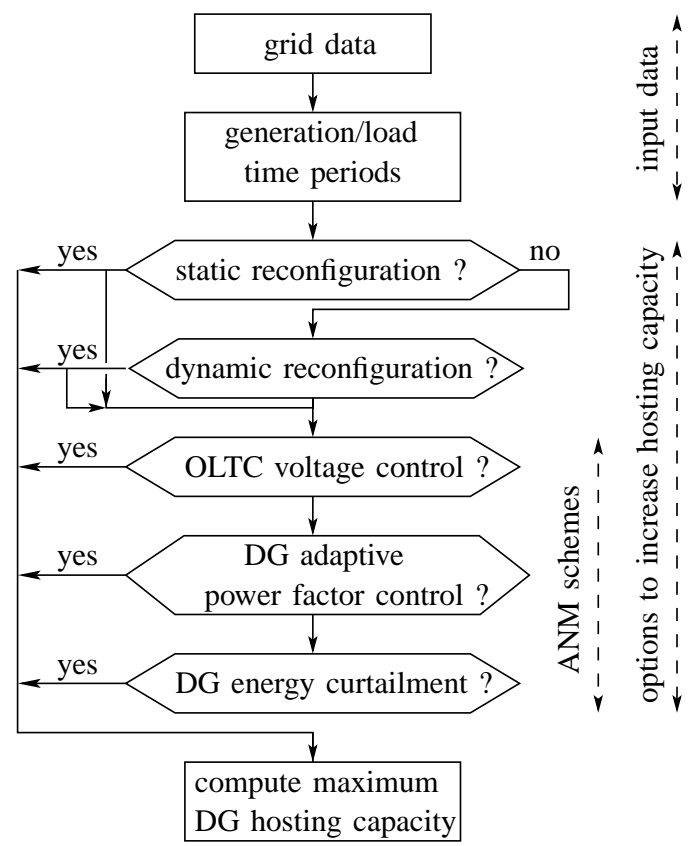

Fig. 1. Overview of the proposed method

essential in order to render the problem manageable and reduce the computational burden of the MINLP MP-OPF problem. Here, this is done by identifying the potentially binding (or critical) periods which actually trigger network constraints.

Network reconfiguration (both static and dynamic) is investigated using the following iterative algorithm:

1) Initialize the set of potentially binding periods by selecting only the worst-case demand/generation scenario.

2) Solve the MP-OPF model (1)-(14) by considering only the current set of potentially binding periods.

Note that if dynamic reconfiguration is allowed, it is assumed that the network topology change in a given period impacts all subsequent periods, which keep therefore the same topology, as long as a new topology change in another period occurs ${ }^{1}$.

3) At the solution of the problem (i.e., new topology and DG nominal capacity) check, using a classical power flow program, whether there are operational constraints violations in the remaining periods.

If the operational constraints of any period are satisfied, then an acceptable solution of the MP-OPF problem is obtained.

Note that if dynamic reconfiguration is allowed the feasibility of every period $m$ which presents violated constraints is further individually checked by a special

\footnotetext{
${ }^{1}$ Assume for instance the following set of $k$ periods $\left\{m_{1}, m_{2}, m_{3}, \ldots, m_{i-1}, m_{i}, \ldots, m_{k}\right\}$ and that topology changes occur in periods $m_{3}$ and $m_{i}$. According to the periods processing in dynamic reconfiguration, the grid operates in three different topologies between periods: $m_{1}$ to $m_{2}$ (the original topology), $m_{3}$ to $m_{i-1}$, and $m_{i}$ to $m_{k}$, respectively.
} 
case of MP-OPF model, which is formulated as follows:

$$
\begin{array}{ll}
\min & \sum_{i j \in L} r_{i j}+\sum_{i \in N}\left(r_{i \min }+r_{i \max }\right) \\
\text { s.t. } & (2)-(5),(9),(13),(14) \\
& \left(I_{i j}^{m}\right)^{2} \leq K_{I}\left(1-s_{i j}^{m}\right)+s_{i j}^{m}\left(I_{i j}^{\max }\right)^{2}+r_{i j}, i j \in L \\
& V_{i \min }^{2}-r_{i \min } \leq\left(e_{i}^{m}\right)^{2}+\left(f_{i}^{m}\right)^{2}, i \in N \\
& \left(e_{i}^{m}\right)^{2}+\left(f_{i}^{m}\right)^{2} \leq V_{i \max }^{2}+r_{i \max }, i \in N \\
& r_{i \min }, r_{i \max } \geq 0, i \in N \\
& r_{i j} \geq 0, i j \in L
\end{array}
$$

In this formulation constraints (17), (18), and (19) are relaxations of original operational constraints (6) and (7), obtained by means of additional non-negative continuous variables $r_{i j}, r_{i \text { min }}, r_{i \text { max }}$. This optimization problem looks for optimal switching actions on RCS that minimizes the degree of relaxation (or infeasibility) of the problem.

Note that if dynamic reconfiguration is needed to remove violated constraints in a given period, the grid topology is switched back in the next period to the safe topology computed by MP-OPF in step 2, since the new topology resulted after dynamic reconfiguration may not ensure feasibility of subsequent periods.

4) For periods which lead to violated operational constraints build up three period rankings according to the maximum violation of: lower voltage limit, upper voltage limit, and thermal limit, respectively. Pick up the top period ${ }^{2}$ from each ranking and add it to the set of potentially binding periods.

Go to step 2.

Although not described explicitly, the ANM schemes can be incorporated straightforwardly in this algorithm.

\section{CASE STUdY}

In this section, different cases involving potential DG sites, reconfiguration strategies and the use of the ANM schemes are studied considering a modified benchmark distribution system. First, the network's maximum hosting capacity (MHC) is assessed adopting static reconfiguration. Then, the benefit of dynamic reconfiguration as a single ANM scheme is assessed. The combined benefits of static reconfiguration and classical ANM schemes are explored next. Finally, the computational aspects of the proposed technique for reducing the problem size are discussed.

\section{A. Test system}

The proposed approach is applied on the widely used 34-bus $12.66 \mathrm{kV}$ distribution system [18], see Fig. 2. This benchmark system was modified to consider eight potential sites for DG.

\footnotetext{
${ }^{2}$ Note that if DG curtailment option is allowed, the curtailment may spread over a significant number of periods, depending on the value of parameter $\gamma$. Consequently, the algorithm performance requires further tuning regarding the choice of the number of periods to be selected for inclusion into the MP-OPF problem.
}

TABLE I

CHARACTERISTICS OF TEST DISTRIBUTION SYSTEM

\begin{tabular}{|c|c|c|c|c|c|}
\hline nodes & lines & $\begin{array}{c}\text { sectionalizing } \\
\text { switches }\end{array}$ & $\begin{array}{c}\text { tie } \\
\text { switches }\end{array}$ & feeders & $\begin{array}{c}\text { potential } \\
\text { DG sites }\end{array}$ \\
\hline \hline 34 & 37 & 31 & 5 & 1 & 8 \\
\hline
\end{tabular}

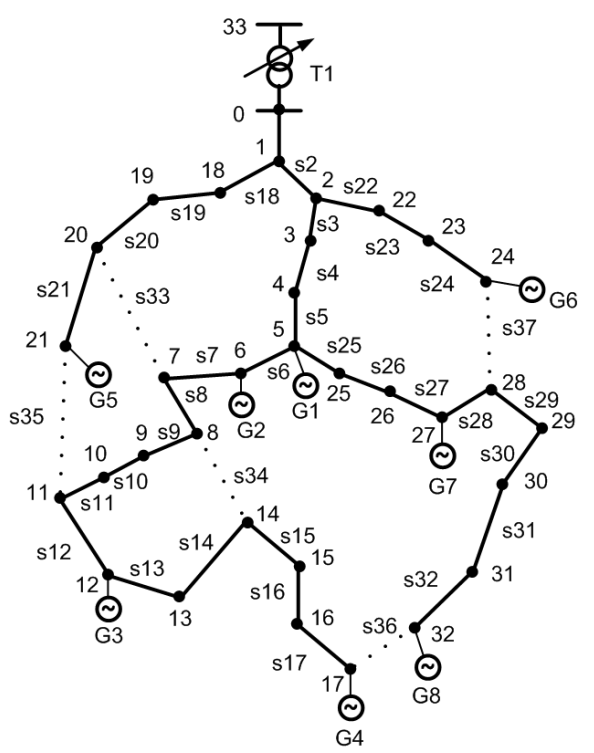

Fig. 2. One line diagram of the modified 34-bus distribution grid [18].

A summary of the characteristics of the test system is given in Table I. In the business as usual network operation, the normally closed switches (s2 to s32) are called sectionalizing switches, and the normally open switches (s33 to s37) are called tie (or emergency) switches.

The peak load is 3.715 MW and 2.3 MVar. The minimum load is $40 \%$ of the peak. Eight potential sites for the deployment of DG units (G1 to G8) are considered as shown in Fig. 2. A mix of locations (mid and end points of feeders) for these generators were chosen in order to mimic different types of connections.

\section{B. Considerations}

Unless otherwise specified, a set of 146 (aggregated) load periods and two wind profiles (WP1 and WP2) from [9], and shown in Fig. 3, are considered (i.e., historic demand and wind data corresponds to central Scotland in 2003). Generators G1, G2, G7, and G8 are assumed to follow WP1 whereas G3, G4, G5, and G6 are assumed to behave according to WP2.

Three cases for the deployment of DG are considered:

- Case A: only two sites are available (G5 and G8);

- Case B: only six sites are available (G1, G2, G3, G4, G7, and G8);

- Case C: all eight sites (G1 to G8) are available.

The minimum and maximum voltage limits are set to 0.95 p.u. and 1.05 p.u. at all nodes, respectively, aligned with MV limits common in Europe. The voltage controlled by the OLTC at bus 0 has a deadband ${ }^{3}$ of 1.02 p.u. to 1.045 p.u.. The thermal

\footnotetext{
${ }^{3}$ The lower bound has been chosen so that to ensure that all voltages in all periods are above minimum limit.
} 


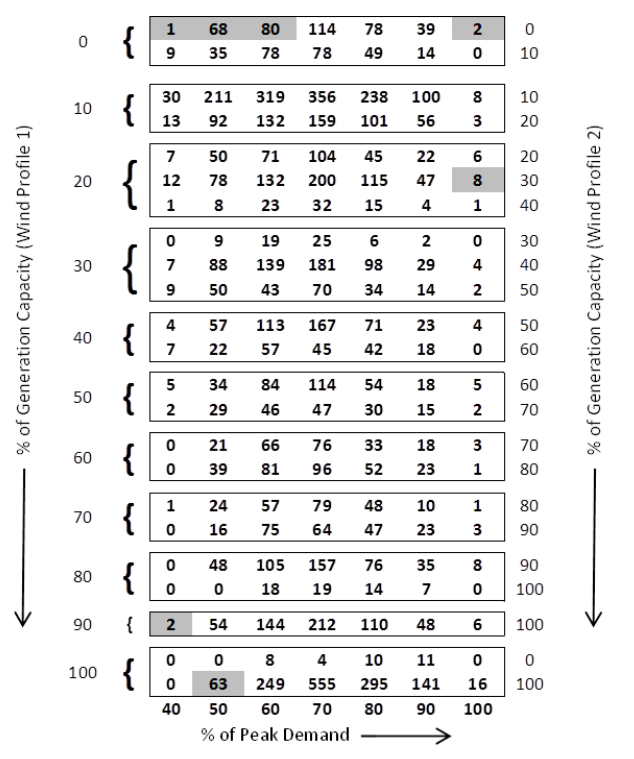

Fig. 3. Number of coincident hours during one year for 146 aggregated demand/generation periods and two different wind sites [9]. The shaded cells indicate the set of periods which have been found binding in the optimization problems solved in the paper.

TABLE II

ORIGINAL CONFIGURATION: NOMINAL CAPACITY OF DG UNITS (MW) AND MAXIMUM HOSTING CAPACITY (MHC)

\begin{tabular}{|c|c|c|c|}
\hline DG unit & case A & case B & case C \\
\hline \hline G1 & - & 2.082 & 2.991 \\
\hline G2 & - & 1.135 & 0.0 \\
\hline G3 & - & 0.0 & 0.0 \\
\hline G4 & - & 0.257 & 0.0 \\
\hline G5 & 1.924 & - & 1.809 \\
\hline G6 & - & - & 1.744 \\
\hline G7 & - & 0.285 & 0.189 \\
\hline G8 & 1.698 & 0.402 & 0.420 \\
\hline \hline$M H C$ & 3.622 & 4.161 & 7.154 \\
\hline
\end{tabular}

limit of all lines is set to 6.6 MVA (which corresponds to a current of $300 \mathrm{~A}$ ).

The MINLP optimization model has been developed in GAMS version 23.9.3 [35] and is solved using the simple branch and bound solver. All tests have been performed on a PC of 2.8-GHz and 4-Gb RAM.

\section{MHC for the original configuration}

For benchmarking purposes, the network's maximum hosting capacity is quantified for the original configuration (see Fig. 2) and the three cases of available DG sites. The original configuration is taken as that without any reconfiguration capabilities, i.e., passive grid management. The results are shown in Table II.

As expected the largest hosting capacity is obtained in case $\mathrm{C}$ due to the larger number of degrees of freedom for DG location than in cases A and B. Note that, in cases B and $\mathrm{C}$, the nominal DG capacity at some locations is zero, which is a natural consequence of the linear objective (1). As voltage rise is the limiting constraint in all cases, results also confirm the principle that the closer the DG location is to the
TABLE III

STATIC GRID RECONFIGURATION ONLY: NOMINAL CAPACITY OF DG UNITS (MW) AND MAXIMUM HOSTING CAPACITY (MHC)

\begin{tabular}{|c|c|c|c|c|c|c|c|}
\hline \multirow{2}{*}{ DG unit } & \multicolumn{2}{|c|}{ case A } & \multicolumn{2}{c|}{ case B } & \multicolumn{3}{|c|}{ case C } \\
\cline { 2 - 8 } & it. 1 & it. 2 & it. 1 & it. 2 & it. 1 & it. 2 & it.3 \\
\hline \hline G1 & - & - & 2.457 & $\mathbf{2 . 2 1 6}$ & 2.280 & 2.471 & $\mathbf{2 . 8 3 0}$ \\
\hline G2 & - & - & 0.889 & $\mathbf{0 . 8 6 5}$ & 0.555 & 0.8 & $\mathbf{0 . 6 1 1}$ \\
\hline G3 & - & - & 0.716 & $\mathbf{0 . 7 9 4}$ & 0.0 & 0.0 & $\mathbf{0 . 0}$ \\
\hline G4 & - & - & 0.365 & $\mathbf{0 . 3 1 8}$ & 0.0 & 0.0 & $\mathbf{0 . 0}$ \\
\hline G5 & 1.964 & $\mathbf{2 . 5 1 9}$ & - & - & 2.163 & 1.751 & $\mathbf{1 . 6 6 6}$ \\
\hline G6 & - & - & - & - & 1.751 & 1.747 & $\mathbf{1 . 7 6 2}$ \\
\hline G7 & - & - & 1.320 & $\mathbf{1 . 3 6 1}$ & 0.0 & 0.0 & $\mathbf{0 . 0}$ \\
\hline G8 & 2.666 & $\mathbf{2 . 0 4 4}$ & 0.746 & $\mathbf{0 . 7 6 5}$ & 0.696 & 0.486 & $\mathbf{0 . 3 5 9}$ \\
\hline \hline$M H C$ & 4.630 & $\mathbf{4 . 5 6 3}$ & 6.493 & $\mathbf{6 . 3 1 9}$ & 7.445 & 7.255 & $\mathbf{7 . 2 2 8}$ \\
\hline gain (\%) & & $\mathbf{2 6 . 0}$ & & $\mathbf{5 1 . 8}$ & & & $\mathbf{1 . 0}$ \\
\hline
\end{tabular}

substation, the larger the nominal capacity. Indeed, G1 is the most convenient DG location in cases $\mathrm{B}$ and $\mathrm{C}$ as it is the closest to the substation.

\section{MHC using static reconfiguration}

This study aims to analyze, using the iterative algorithm described in Section III, the impact of static reconfiguration on hosting capacity without ANM schemes. The static reconfiguration approach means that a new feasible configuration to be used during normal operation will be searched in order to increase the MHC of the network.

It is important to emphasize that the MINLP MP-OPF problem including all 146 periods cannot be solved to optimality due to both computer memory and internal solver limitations. Such limitations are to be expected due to both the very large combinatorial space of the problem (i.e., $2^{36}$ possible states stemming from 36 switches statuses) and large size of each sub-problem (for fixed values of switches status the MP-OPF problem size is roughly equivalent to an OPF problem applied to a 34 nodes $\times 146$ periods $=4,964$ bus system). Therefore, the proposed iterative algorithm is a useful means to breakdown the problem size.

The results at each iteration of the proposed algorithm are gathered in Tables III, IV, and V. Table III reports, in columns that use bold characters, the hosting capacity and the gain compared to the original configuration for the three DG siting cases. Note that, compared to the original configuration (see Table II), the hosting capacity improves significantly in case B $(51.8 \%)$ and case A (26\%), but little in case C (1\%). This suggests that the hosting capacity enhancement highly depends on DG siting and therefore must be assessed on a case by case basis. Note also that as no DG curtailment is in place, the capacity increase translates proportionally into larger energy harvest.

Table IV provides the switching actions in each case. Note that the optimal static reconfiguration requires a reduced number of switching actions (i.e., between two and eight) which requires little implementation effort.

The proposed iterative algorithm is illustrated without loss of generality in case A. First the MINLP MP-OPF optimization problem is solved including only the worst-case scenario (i.e., period 15 in Fig. 3, where only non-zero hours periods are counted from top to down and left to right). For the 
TABLE IV

SWITCHING ACTIONS FOR STATIC GRID RECONFIGURATION

\begin{tabular}{|c|c|c|c|c|c|c|c|}
\hline & \multicolumn{2}{|c|}{ case $\mathrm{A}$} & \multicolumn{2}{|c|}{ case B } & \multicolumn{3}{|c|}{ case C } \\
\hline & it. 1 & \begin{tabular}{|l} 
it. 2 \\
\end{tabular} & it. 1 & it. 2 & it. 1 & it. 2 & it. 3 \\
\hline $\begin{array}{c}\text { open } \\
\text { switches }\end{array}$ & $\begin{array}{l}\mathrm{s} 3, \mathrm{~s} 10 \\
\mathrm{~s} 12, \mathrm{~s} 28\end{array}$ & $\begin{array}{c}\mathbf{s 3 ,}, \mathbf{s 1 3} \\
\text { s26 }\end{array}$ & $\begin{array}{c}\mathrm{s} 6, \mathrm{~s} 9 \\
\mathrm{~s} 13, \mathrm{~s} 26\end{array}$ & $\begin{array}{c}\mathrm{s6}, \mathrm{s9} \\
\mathrm{s} 14, \mathrm{~s} 25\end{array}$ & $\begin{array}{c}\text { s6, s10 } \\
\text { s26 }\end{array}$ & s6 & $\begin{array}{l}s 6 \\
\text { s32 }\end{array}$ \\
\hline $\begin{array}{c}\text { closed } \\
\text { switches }\end{array}$ & $\begin{array}{l}\mathrm{s} 34, \mathrm{~s} 35 \\
\mathrm{~s} 36, \mathrm{~s} 37\end{array}$ & $\begin{array}{c}\mathbf{s 3 5}, \mathbf{s 3 6} \\
\mathbf{s 3 7}\end{array}$ & $\begin{array}{l}\mathrm{s} 33, \mathrm{~s} 34 \\
\mathrm{~s} 35, \mathrm{~s} 37\end{array}$ & $\begin{array}{l}\mathbf{s 3 3}, \mathbf{s 3 4} \\
\mathrm{s} 35, \mathrm{~s} 7\end{array}$ & $\begin{array}{c}\mathrm{s} 33, \mathrm{~s} 35 \\
\mathrm{~s} 36\end{array}$ & s33 & $\begin{array}{l}\mathbf{s 3 3} \\
\text { s36 }\end{array}$ \\
\hline
\end{tabular}

TABLE $\mathrm{V}$

STATIC GRID RECONFIGURATION: PERIODS CONSIDERED

\begin{tabular}{|c|c|c|c|c|c|c|}
\hline \multicolumn{2}{|r|}{ case A } & \multicolumn{2}{|c|}{ case B } & \multicolumn{3}{|c|}{ case $\mathrm{C}$} \\
\hline it. 1 & it. 2 & it. 1 & it. 2 & it. 1 & it. 2 & it. 3 \\
\hline 15 & $15,129,134$ & 15 & 15,36 & 15 & 15,129 & $15,129,36$ \\
\hline
\end{tabular}

obtained new grid topology and DG nominal capacity the remaining 145 periods are checked for operational constraint violation. Voltage limits are violated in most periods. Periods 129 and 134 are selected to be included into optimization problem as they lead to the worst upper and lower voltage limits violation, respectively. The MINLP MP-OPF optimization problem including these three critical periods is solved again and provides a new static topology and DG nominal capacity. As for this new solution no constraint is violated in any period, the optimal solution is found and the algorithm terminates. Table $\mathrm{V}$ yields the periods added successively into optimization. Critical periods on both static and dynamic reconfiguration are shaded in Fig. 3.

From the results reported in these tables the following observations can be made:

- in all cases only 2-3 periods out of 146 are critical and almost the same periods are included in the optimization in every case (see Table V);

- the optimal solution does not correspond only to the worst-case period (see column labeled "it. 1" in Table III) but is strongly influenced by it;

- the algorithm needs a small number of (external) iterations to converge, i.e., 2 loops in cases $\mathrm{A}$ and $\mathrm{B}$, and 3 loops in case $\mathrm{C}$.

Table VI reports the hosting capacity and the gain compared to the original configuration for the three DG siting cases and three values of maximum allowed number of static reconfiguration switching actions $\Delta s^{s t a}$ (where $\Delta s^{s t a}=36$ is the maximum). Table VII provides the switching actions in each case. What is very important from this analysis is that a small number of switching actions (e.g., 4 actions) is almost as effective as the case with unlimited number of switching actions.

\section{E. MHC using dynamic reconfiguration}

Here the impact on the hosting capacity from dynamic reconfiguration, as a single ANM scheme, will be assessed. To investigate the benefits of dynamic reconfiguration, in the context of future active/smart grids, two sets of 18 and 9 remotely controlled switches (RCS) are considered.

The first RCS set comprises the 5 tie switches (s33, s34, s35, s36, and s37) and 13 sectionalizing switches placed at
TABLE VI

STATIC GRID RECONFIGURATION ONLY: NOMINAL CAPACITY OF DG UNITS (MW) AND MAXIMUM HOSTING CAPACITY (MHC)

\begin{tabular}{|c|c|c|c|c|c|c|c|c|c|}
\hline \multirow{2}{*}{$\begin{array}{c}\text { DG } \\
\text { unit }\end{array}$} & \multicolumn{3}{|c|}{ case A } & \multicolumn{3}{c|}{ case B } & \multicolumn{3}{c|}{ case C } \\
\cline { 2 - 10 } & 2 & 4 & 36 & 2 & 4 & 36 & 2 & 4 & 36 \\
\hline \hline G1 & - & - & - & 1.584 & 2.228 & 2.216 & 2.353 & 2.353 & 2.830 \\
\hline G2 & - & - & - & 1.403 & 1.026 & 0.865 & 0.506 & 0.506 & 0.611 \\
\hline G3 & - & - & - & 0.0 & 0.635 & 0.794 & 0.439 & 0.439 & 0.0 \\
\hline G4 & - & - & - & 0.0 & 0.0 & 0.318 & 0.0 & 0.0 & 0.0 \\
\hline G5 & 2.576 & 2.704 & 2.519 & - & - & - & 1.168 & 1.168 & 1.666 \\
\hline G6 & - & - & - & - & - & - & 1.760 & 1.760 & 1.762 \\
\hline G7 & - & - & - & 1.382 & 1.364 & 1.361 & 0.0 & 0.0 & 0.0 \\
\hline G8 & 1.477 & 1.768 & 2.563 & 0.760 & 0.768 & 0.765 & 0.522 & 0.522 & 0.359 \\
\hline \hline$M H C$ & 4.053 & 4.472 & 4.563 & 5.129 & 6.021 & 6.319 & 7.198 & 7.198 & 7.228 \\
\hline gain (\%) & 11.9 & 23.4 & 26.0 & 23.3 & 44.7 & 51.8 & 0.6 & 0.6 & 1.0 \\
\hline
\end{tabular}

TABLE VII

STATIC GRID RECONFIGURATION WITH LIMITED NUMBER OF SWITCHING ACTIONS

\begin{tabular}{|c|c|c|c|c|c|c|}
\hline switches & \multicolumn{2}{|c|}{ case $\mathrm{A}, \Delta s^{\text {sta }}=$} & \multicolumn{2}{|c|}{ case $\mathrm{B}, \Delta s^{\text {sta }}=$} & \multicolumn{2}{c|}{ case $\mathrm{C}, \Delta s^{\text {sta }}=$} \\
& 2 & 4 & 2 & 4 & 2 & 4 \\
\hline \hline open & $\mathrm{s} 6$ & $\mathrm{~s} 5, \mathrm{~s} 28$ & $\mathrm{~s} 25$ & $\mathrm{~s} 6, \mathrm{~s} 25$ & $\mathrm{~s} 6$ & $\mathrm{~s} 6$ \\
\hline closed & $\mathrm{s} 35$ & $\mathrm{~s} 35, \mathrm{~s} 37$ & $\mathrm{~s} 37$ & $\mathrm{~s} 33, \mathrm{~s} 37$ & $\mathrm{~s} 33$ & $\mathrm{~s} 33$ \\
\hline
\end{tabular}

key locations in the network, namely s2, s4, s6, s7, s10, s12, s14, s16, s20, s21, s23, s27, and s31.

Table VIII reports the hosting capacity and the gain compared to the original configuration for the three DG siting cases and different values of $\Delta s^{d y n}$, the maximum allowed number of switching actions on RCS from one state to another. Table IX provides the switching actions in each case.

By comparing Tables VI and VIII one can conclude that the hosting capacity increase due to static and dynamic reconfiguration is almost equal (the largest difference is of $3.6 \%$ in case C). However, if only two switching actions are allowed, the dynamic reconfiguration outperforms the static one in all cases (the largest difference is of $21.6 \%$ in case B). It also provides MHC only slightly lower than the best MHC values (the largest difference if of $7.2 \%$ in case B).

Note that, although 18 RCS evenly distributed in the network are available, only few switching actions are needed to reach the optimum (4 actions in cases $\mathrm{A}$ and $\mathrm{B}$, and 6 actions in case C). Observe also that the larger the number of switching actions, the larger the number of critical periods in which switching actions are performed. The number of critical periods is 6 , while only 4 periods are critical for static reconfiguration (see Table $\mathrm{V}$ ).

The second set of RCS is a sub-set of the former and comprises the 5 tie-switches (s33, s34, s35, s36, s37) and 4 well located sectionalizing switches (s7, s20, s23, s27). Table X provides the hosting capacity in the three cases. Observe that 2 switching actions are sufficient to maximize the hosting capacity. By comparing the MHC from this table with that of Table VIII one can notice that the MHC increase is only slightly inferior to that obtained with the larger set of 18 RCS (the largest difference is of $5.8 \%$ in case A). This proves that a limited number of well located RCS can efficiently increase the MHC.

Consequently, if a sufficient number of RCS are deployed 
TABLE VIII

DYNAMIC GRID RECONFIGURATION ONLY: NOMINAL CAPACITY OF DG UNITS (MW) AND MAXIMUM HOSTING CAPACITY (MHC)

\begin{tabular}{|c|c|c|c|c|c|c|c|}
\hline \multirow{2}{*}{$\begin{array}{c}\text { DG } \\
\text { unit }\end{array}$} & \multicolumn{2}{|c|}{ case A } & \multicolumn{2}{c|}{ case B } & \multicolumn{3}{c|}{ case C } \\
\cline { 2 - 8 } & $=2$ & $\in s^{\text {dyn }}$ & \multicolumn{2}{c|}{$\Delta s^{\text {dyn }}$} & \multicolumn{3}{c|}{$\Delta s^{\text {dyn }}$} \\
\hline \hline G1 & - & - & 2.306 & 2.295 & 2.200 & 2.316 & 2.315 \\
\hline G2 & - & - & 1.026 & 1.006 & 0.236 & 0.643 & 0.555 \\
\hline G3 & - & - & 0.635 & 0.862 & 0.0 & 0.0 & 0.0 \\
\hline G4 & - & - & 0.0 & 0.078 & 0.0 & 0.0 & 0.0 \\
\hline G5 & 2.708 & 2.717 & - & - & 2.181 & 1.685 & 1.887 \\
\hline G6 & - & - & - & - & 1.751 & 1.751 & 1.750 \\
\hline G7 & - & - & 1.275 & 1.305 & 0.0 & 0.0 & 0.359 \\
\hline G8 & 1.785 & 1.785 & 0.776 & 0.755 & 1.032 & 1.062 & 0.620 \\
\hline \hline$M H C$ & 4.493 & 4.502 & 6.018 & 6.251 & 7.400 & 7.457 & 7.483 \\
\hline gain (\%) & 24.0 & 24.1 & 44.6 & 50.2 & 3.4 & 4.2 & 4.6 \\
\hline
\end{tabular}

TABLE IX

SWITCHING ACTIONS FOR DYNAMIC GRID RECONFIGURATION

\begin{tabular}{|c|c|c|c|c|c|c|}
\hline & case & $\overline{\mathrm{A}, \Delta s^{d y n}}$ & $=2$ & & & \\
\hline critical periods & 1 & 15 & 16 & 36 & 37 & 129 \\
\hline open switches & s4 & s27 & - & - & - & - \\
\hline closed switches & $\mathrm{s} 35$ & s37 & - & - & - & - \\
\hline & case $\mathrm{A}$, & $4 \leq \Delta s^{d y}$ & $\leq 18$ & & & \\
\hline critical periods & 1 & 15 & 16 & 36 & 37 & 129 \\
\hline open switches & s27 & $\mathrm{s} 4, \mathrm{~s} 10$ & $\overline{\text { s14 }}$ & - & - & s7 \\
\hline closed switches & s37 & s34, s35 & s10 & - & - & s27 \\
\hline & case & $3, \Delta s^{d y n}$ & & & & \\
\hline critical periods & 1 & 15 & 16 & 36 & 37 & 129 \\
\hline open switches & s6 & s27 & - & - & - & - \\
\hline closed switches & $\mathrm{s} 33$ & s37 & - & - & - & - \\
\hline & case $\mathrm{B}$, & $4 \leq \Delta s^{d y}$ & $\leq 18$ & & & \\
\hline critical periods & 1 & 15 & 16 & 36 & 37 & 129 \\
\hline open switches & $\overline{\text { s6 }}$ & s10, s27 & $\overline{\mathrm{s} 16}$ & $=$ & $\overline{-1}$ & - \\
\hline closed switches & s35 & s33, s37 & $\mathrm{s} 36$ & - & - & - \\
\hline & case & $\overline{\mathrm{C}, \Delta s^{d y n}}$ & & & & \\
\hline critical periods & 1 & 15 & 16 & 36 & 37 & 129 \\
\hline open switches & s7 & s27 & s36 & - & - & - \\
\hline closed switches & s36 & $\mathrm{s} 35$ & s27 & - & - & - \\
\hline & case & $\overline{\mathrm{C}, \Delta s^{d y n}}$ & & & & \\
\hline critical periods & 1 & 15 & 16 & 36 & 37 & 129 \\
\hline open switches & s14, s16 & s6, s27 & s7, s12 & s6 & s36 & - \\
\hline closed switches & $\mathrm{s} 34, \mathrm{~s} 36$ & $\mathrm{~s} 16, \mathrm{~s} 33$ & s6, s14 & s7 & s27 & - \\
\hline & case $\mathrm{C}$, & $5 \leq \Delta s^{d y}$ & $\leq 18$ & & & \\
\hline critical periods & 1 & 15 & 16 & 36 & 37 & 129 \\
\hline open switches & s6, s27, s31 & s10, s37 & s7 & s23, s31 & - & - \\
\hline closed switches & $\mathrm{s} 35, \mathrm{~s} 36, \mathrm{~s} 37$ & $\mathrm{~s} 31, \mathrm{~s} 33$ & s6 & s27, s37 & - & - \\
\hline
\end{tabular}

TABLE $X$

DYNAMIC GRID RECONFIGURATION ONLY: NOMINAL CAPACITY OF DG UNITS (MW) AND MAXIMUM HOSTING CAPACITY (MHC)

\begin{tabular}{|c|c|c|c|}
\hline DG & \multicolumn{3}{|c|}{$2 \leq \Delta s^{\text {dyn }} \leq 9$} \\
unit & case A & case B & case C \\
\hline \hline G1 & - & 2.395 & 2.200 \\
\hline G2 & - & 0.236 & 0.236 \\
\hline G3 & - & 1.176 & 0.0 \\
\hline G4 & - & 0.115 & 0.0 \\
\hline G5 & 1.903 & - & 2.181 \\
\hline G6 & - & - & 1.751 \\
\hline G7 & - & 1.290 & 0.0 \\
\hline G8 & 2.381 & 0.749 & 1.032 \\
\hline \hline$M H C$ & 4.284 & 5.961 & 7.400 \\
\hline gain (\%) & 18.3 & 43.3 & 3.4 \\
\hline
\end{tabular}

at key locations in the network, it is expected that, over the whole set of periods considered, the dynamic reconfiguration has a better performance in terms of hosting capacity than the static approach. This is due to its ability to adapt to changing operating conditions. However, on the other hand, since the RCS actions tend to be triggered often, the dynamic approach has some drawbacks such as the wear and tear cost of the switching actions and the risk of failure.

As, in this particular case study, the use of dynamic reconfiguration does not bring a significantly larger improvement over the static reconfiguration, only the latter is investigated hereafter.

\section{F. MHC using static reconfiguration and ANM schemes}

This subsection focuses on the benefits on hosting capacity from static reconfiguration (SR) in the presence of ANM schemes such as OLTC-based voltage control (VC), adaptive power factor control (APFC), and energy curtailment (EC).

The VC scheme assumes that the secondary voltage of the OLTC transformer can be centrally controlled to operate outside original deadband (i.e., 1.02 p.u. to 1.045 p.u.) and adopts the network voltage limits of 0.95 p.u. to 1.05 p.u. The APFC scheme assumes that the power factor of each DG unit can be controlled within the range 0.95 lagging to 0.95 leading. The EC scheme assumes a percentage of allowed curtailed energy from the total energy of $5 \%$, i.e., the original capacity factor will be affected by $5 \%$ at most.

Table XI reports the results obtained for the three DG siting cases and various combinations of control variables in the ANM scheme and static reconfiguration. Cases A0, B0, C0 correspond to the hosting capacity in the original configuration without static reconfiguration or ANM schemes. The following observations can be made:

- among all options taken individually, the VC ANM scheme (i.e., cases A2, B2, C2) leads to the largest improvement of the hosting capacity in all cases;

- the APFC ANM scheme alone (i.e., cases A3, B3, C3) leads to a significant improvement of the hosting capacity but is however less efficient than the VC scheme;

- the combination of VC and APFC (i.e., cases A4, B4, C4) improves very little the MHC, except for case A4;

- as found in other works [9], including EC leads to a substantial increase of MHC in all cases (e.g., $43.6 \%$ between cases B5 and B4). In particular in case C, where the grid capacity is "saturated" due to the even distribution of DG and hence the alternative options are less effective in enhancing hosting capacity than in cases $\mathrm{A}$ and $\mathrm{B}$, the EC option doubles the MHC (e.g., case C5 vs. case C4).

- considering static reconfiguration jointly with other ANM schemes (e.g., cases A7, B7, C7) increases the MHC with up to $36.7 \%$ (e.g., case B5 vs. case B7) compared to the ANM schemes (e.g., cases A5, B5, C5).

Although the static reconfiguration option is not as effective as the ANM schemes, it leads to a considerable increase of the grid capacity of up to $51.8 \%$. Furthermore, it has a low cost and can be readily implemented. These are significant 
TABLE XI

MHC (MW) FOR VARIOUS COMBINATIONS OF STATIC RECONFIGURATION (SR) AND ANM SCHEMES

\begin{tabular}{|c|c|c|c|c|c|c|}
\hline case & VC & APFC & EC & SR & MHC & gain (\%) \\
\hline \hline A0 & - & - & - & - & 3.622 & - \\
\hline A1 & - & - & - & $x$ & 4.563 & 26.0 \\
\hline A2 & $x$ & - & - & - & 6.422 & 77.3 \\
\hline A3 & - & $x$ & - & - & 5.526 & 52.5 \\
\hline A4 & $x$ & $x$ & - & - & 8.803 & 143.0 \\
\hline A5 & $x$ & $x$ & $x$ & - & 10.484 & 189.4 \\
\hline A6 & $x$ & $x$ & - & $x$ & 8.983 & 148.0 \\
\hline A7 & $x$ & $x$ & $x$ & $x$ & 11.066 & 205.5 \\
\hline \hline B0 & - & - & - & - & 4.161 & - \\
\hline B1 & - & - & - & $x$ & 6.319 & 51.8 \\
\hline B2 & $x$ & - & - & - & 7.924 & 90.4 \\
\hline B3 & - & $x$ & - & - & 5.581 & 34.1 \\
\hline B4 & $x$ & $x$ & - & - & 7.981 & 91.8 \\
\hline B5 & $x$ & $x$ & $x$ & - & 9.747 & 134.2 \\
\hline B6 & $x$ & $x$ & - & $x$ & 8.975 & 115.7 \\
\hline B7 & $x$ & $x$ & $x$ & $x$ & 11.272 & 170.9 \\
\hline \hline C0 & - & - & - & - & 7.154 & - \\
\hline C1 & - & - & - & $x$ & 7.228 & 1.0 \\
\hline C2 & $x$ & - & - & - & 8.688 & 21.4 \\
\hline C3 & - & $x$ & - & - & 8.439 & 19.3 \\
\hline C4 & $x$ & $x$ & - & - & 8.846 & 23.7 \\
\hline C5 & $x$ & $x$ & $x$ & - & 11.200 & 56.5 \\
\hline C6 & $x$ & $x$ & - & $x$ & 8.979 & 25.5 \\
\hline C7 & $x$ & $x$ & $x$ & $x$ & 11.200 & 56.5 \\
\hline
\end{tabular}

TABLE XII

RANGE OF COMPUTATIONAL TIME (S)

\begin{tabular}{|c|c|}
\hline case & static reconfiguration \\
\hline \hline A & $565 \ldots 8,127$ \\
\hline B & $469 \ldots 7,213$ \\
\hline C & $603 \ldots 9,926$ \\
\hline \hline case & dynamic reconfiguration \\
\hline \hline A & $850 \ldots 13,884$ \\
\hline B & $615 \ldots 9,377$ \\
\hline C & $702 \ldots 14,291$ \\
\hline \hline case & ANM schemes and/or static reconfiguration \\
\hline \hline A1, B1, C1 & $469 \ldots 7,213$ \\
\hline A2, B2, C2 & $27 \ldots 48$ \\
\hline A3, B3, C3 & $32 \ldots 46$ \\
\hline A4, B4, C4 & $41 \ldots 53$ \\
\hline A5, B5, C5 & $49 \ldots 61$ \\
\hline A6, B6, C6 & $589 \ldots 8,249$ \\
\hline A7, B7, C7 & $727 \ldots 10,131$ \\
\hline
\end{tabular}

advantages over alternative ANM schemes which require communication infrastructure or result in tear and wear cost (e.g., OLTC operation in the VC option).

\section{G. Computational performance}

Table XII provides the range of computational time obtained in the different cases investigated: static reconfiguration (Table VI), dynamic reconfiguration (Table VIII) and various combinations of ANM schemes and grid reconfiguration (Table XI).

From this table it can be observed that, when only continuous variables (i.e., ANM options) are considered in the optimization, computational times range from a few tens of seconds to a few minutes. On the other hand, when only binary variables (i.e., for static or dynamic reconfiguration) are used in the optimization, the computational effort increases significantly to a few tens of minutes to several hours. Compared to the latter case the computational burden increases little when both continuous and binary variables are optimized together.

\section{Discussion}

The approaches investigated in this paper are for planning purposes and consequently their solution time scales can be considered acceptable. In addition, it is important to highlight that to assess the corresponding performance the case study considered a significant hypothetical number of switches. In practice, distribution networks do not have a large number of reconfiguration options due to operational or safety aspects. This reduces significantly the computational burden. Further reduction of the computational effort can be obtained by various means such as: parallelization of MINLP algorithms, use of more powerful computer architecture, network model reduction using network equivalents, etc. Consequently, the proposed approach could potentially be used in large real-life distribution networks.

This work considers a single optimization objective and is formulated as a MINLP problem. Other optimization techniques such as meta-heuristics [17], [20], [21], [31] can also be adopted. In particular, the approach could be extended to consider the trade-off among different operational aspects such as losses, reliability, number of switching actions, etc. [3], [4].

The proposed method has been illustrated for a set of multiperiods (i.e., demand and two wind profiles) recorded in realworld during one year [9]. However, the method is generic and can incorporate samples from any kind of renewable energy source. As the computed grid hosting capacity depends on such input data, it is acknowledged that it is very challenging to define properly those periods given the difficulties with obtaining good forecast for some technologies even for the next 24 hours. Therefore the method should be extended to incorporate uncertainty related to renewable energy sources. The method can be also used to trade-off between static and dynamic reconfiguration with respect to seasonal changes in renewables output. Nevertheless, the ultimate goal of our approach is to provide a way to assess the grid capacity and thereby to aid planning investments in grid reinforcement.

The DG curtailment approach adopted in the paper is simple yet it provides a very good idea of how far DG penetrations can go if each DG operator allows the same level of curtailment. Other approaches, however, can certainly be implemented in the methodology once defined [34].

Due to the planning focus of the proposed method, dynamic reconfiguration operational aspects such as protection or the interaction with network elements (e.g., capacitor banks) have not been considered. This, however, can be implemented for instance by taking into account fault level constraints [8] as well as detailed models of network elements.

Finally, it is important to recognize that the proposed dynamic reconfiguration algorithm, although for planning purposes, assumes that in practice the responses are rapid. Hence, for operational analyses, the information obtained from remote controlled equipment and other sensors (such as smart meters) should be considered. 


\section{CONCLUSIONS}

This paper proposes a multi-period OPF approach for assessing the improvement of DG hosting capacity of distribution systems by applying static reconfiguration or dynamic reconfiguration, together with active network management schemes.

Results show that the application of static or dynamic reconfiguration is an effective means to accommodate larger amounts of DG in distribution systems without network reinforcement. Furthermore, another noteworthy result for the studied benchmark network is that the optimal topology requires a limited number of switching actions (e.g., 6 to 8 actions) which facilitates its implementation. In particular a very small number of switching actions (e.g., 2 to 4 ) is almost as effective as the optimal solution.

The static reconfiguration is an efficient available solution that can be used to assess and improve the hosting capacity independently of the more complex and CAPEX intensive ANM schemes, e.g., as an intermediate step between currently passive and future active distribution systems.

The effectiveness of dynamic reconfiguration heavily depends on the number and deployment of available remotely controlled switches. It can lead to larger DG hosting capacity than static reconfiguration due to its ability to adapt to changing operating conditions. However, since the remotely controlled switching actions tend to be triggered often, the dynamic approach has some drawbacks such as the wear and tear cost of the switching actions and the risk of failure.

\section{REFERENCES}

[1] M.J. Dolan, E.M. Davidson, I. Kockar, G.W. Ault, and S.D.J. McArthur, "Distribution power flow management utilizing an online optimal power flow technique", IEEE Trans. on Power Syst., Vol. 27, No. 2, pp. 790799, May 2012.

[2] T. Sansawatt, L.F. Ochoa, and G.P. Harrison, "Smart decentralized control of DG for voltage and thermal constraint management", IEEE Trans. on Power Syst., vol. 27, no. 3, pp. 1637-1645, 2012.

[3] A. Keane, L.F. Ochoa, C.L.T. Borges, G.W. Ault, A. D. AlarconRodriguez, R.A.F. Currie, F. Pilo, C. Dent, and G.P. Harrison, "Stateof-the-Art Techniques and Challenges Ahead for Distributed Generation Planning and Optimization", IEEE Trans. on Power Syst., vol. 28, no. 2, pp. 1493-1502, 2013.

[4] P.S. Georgilakis and N.D. Hatziargyriou, "Optimal Distributed Generation Placement in Power Distribution Networks: Models, Methods, and Future Research", IEEE Trans. on Power Syst., vol. 28, no. 3, pp. 34203428, 2013.

[5] A. Alarcon-Rodriguez, G. W. Ault, and S. J. Galloway, "Multi-objective planning of distributed energy resources: A review of the state-of-theart", Renew. Sustain. Energy Rev., vol. 14, no. 5, pp. 1353-1366, 2010.

[6] S. N. Liew and G. Strbac, "Maximising penetration of wind generation in existing distribution networks", IEE Proc., Gen., Transm. \& Distrib. vol. 149, pp. 256-262, 2002.

[7] A. Keane and M. O'Malley, "Optimal allocation of embedded generation on distribution networks", IEEE Trans. Power Syst., vol. 20, no. 3, pp. 1640-1646, Aug. 2005.

[8] P. Vovos, J. Bialek, "Direct incorporation of fault level constraints in optimal power flow as a tool for network capacity analysis", IEEE Trans. on Power Syst., vol. 20, no. 4, pp. 2125-2134, 2005.

[9] L.F. Ochoa, C. Dent, and G.P. Harrison, "Distribution network capacity assessment: Variable DG and active networks", IEEE Trans. on Power Syst., vol. 25, no. 1, pp. 87-95, 2010.

[10] L.F. Ochoa and G.P. Harrison, "Minimizing energy losses: Optimal accommodation and smart operation of renewable distributed generation", IEEE Trans. Power Syst., vol. 26, no. 1, pp. 198-205, 2011.
[11] Y.M. Atwa, E.F. El-Saadany, M.M.A. Salama, and R. Seethapathy, "Optimal renewable resources mix for distribution system energy loss minimization", IEEE Trans. on Power Syst., vol. 25, no. 1, pp. 360-370, 2010.

[12] G. Celli, E. Ghiani, S. Mocci, and F. Pilo, "A multiobjective evolutionary algorithm for the sizing and siting of distributed generation", IEEE Trans. Power Syst., vol. 20, no. 2, pp. 750-757, 2005.

[13] J.M. Lopez-Lezama, J. Contreras, and A. Padilha-Feltrin, "Location and contract pricing of distributed generation using a genetic algorithm", Int. Journal of Electr. Power Energy Syst., vol. 36, no. 1, pp. 117-126, 2012.

[14] V.F. Martins and C.L.T. Borges, "Active distribution network integrated planning incorporating distributed generation and load response uncertainties", IEEE Trans. Power Syst., vol. 26, no. 4, pp. 2164-2172, 2011.

[15] C. Lueken, P.M.S Carvalho, J. Apt, "Distribution grid reconfiguration reduces power losses and helps integrate renewables", Energy Policy, vol. 48, pp. 260-273, 2012.

[16] P.C. Ramaswamy, P. Vingerhoets, and G. Deconinck, "Reconfiguring distribution grids for more integration of distributed generation", CIRED conference, Stockholm, Sweden, June 2013.

[17] R. Rao, K. Ravindra, K. Satish, and S. Narasimham, "Power loss minimization in distribution system using network reconfiguration in the presence of distributed generation", IEEE Trans. Power Syst., vol. 28 , no. 1 , pp. 317-325, 2013.

[18] M.E. Baran and F.F. Wu, "Network Reconfiguration in Distribution Systems for Loss Reduction and Load Balancing", IEEE Trans. Power Delivery, Vol. 4, No. 2, 1989, pp. 1401-1497.

[19] P.M.S. Carvalho,L.A.F.M. Ferreira, and L.M.F. Barruncho, "Optimization approach to dynamic restoration of distribution systems", Int. Journal of Electrical Power and Energy Systems, vol. 29, no. 3, pp. 222-229, 2007.

[20] A. Botea, J. Rintanen, and D. Banerjee, "Optimal Reconfiguration for Supply Restoration With Informed A* Search”, IEEE Trans. on Smart Grids, vol 3. no. 2, pp. 583-593, 2012.

[21] A. Mendes, N. Boland, P. Guiney, and C. Riveros, "Switch and TapChanger Reconfiguration of Distribution Networks Using Evolutionary Algorithms", IEEE Trans. Power Syst., vol. 28, no. 1, pp. 85-92, 2013.

[22] L.L. Pfitscher, D.P. Bernardon, L.N. Canha, V.F. Montagner, V.J. Garcia, and A.R. Abaide "Intelligent system for automatic reconfiguration of distribution network in real time", Electric Power Systems Research, vol. 97, pp. 84-92, 2013.

[23] M. Lavorato, J.F. Franco, M.J. Rider, and R. Romero, "Imposing Radiality Constraints in Distribution System Optimization Problems", IEEE Trans. on Power Systems, Vol. 27, No. 1, pp. 172-180, 2012.

[24] H.M. Khodr, J. Martinez-Crespo, M.A. Matos, and J. Perreira, "Distribution Systems Reconfiguration Based on OPF Using Benders Decomposition", IEEE Trans. on Power Delivery, vol. 24, no. 4, 2009, pp. 2166-2176.

[25] R. Jabr, R. Singh, and B.C. Pal, "Minimum Loss Network Reconfiguration Using Mixed-Integer Convex Programming" IEEE Trans. on Power Systems, vol. 27, no. 2, 2012, pp. 1106-1115.

[26] J.A. Taylor and F.S. Hover, "Convex Models of Distribution System Reconfiguration" IEEE Trans. Power Syst., vol. 27, no. 3, 2012, pp. 1407-1413.

[27] A. Borghetti, "A Mixed-Integer Linear Programming Approach for the Computation of the Minimum-Losses Radial Configuration of Electrical Distribution Networks", IEEE Trans. Power Syst., vol. 27, no. 3, 2012, pp. 1264-1273.

[28] E. Romero Ramos, J. Riquelme Santos, and J. Reyes, "A simpler and exact mathematical model for the computation of the minimal power losses tree", Electric Power Systems Research, Vol. 80, No. 5, pp. 562571, 2010.

[29] D.P. Bernardon, A.P.C. Mello, L.L. Pfitscher, L.N. Canha, A.R. Abaide, and A.A.B. Ferreira, "Real-time reconfiguration of distribution network with distributed generation", Electric Power Systems Research, vol. 107, pp. 59-67, 2014.

[30] C. Ababei and R. Kavasseri, "Efficient network reconfiguration using minimum cost maximum flow-based branch exchanges and random walks-based loss estimations", IEEE Trans. Power Syst., vol. 26, no. 1, pp. 30-37, 2011.

[31] C. Wang and Y. Gao, "Determination of Power Distribution Network Configuration Using Non-Revisiting Genetic Algorithm”, IEEE Trans. Power Syst., vol. 28, no. 4, 2013, pp. 3638-3648.

[32] F. Pilo, G. Pisano, and G. Soma, "Optimal Coordination of Energy Resources With a Two-Stage Online Active Management", IEEE Trans. on Industrial Electronics, Vol. 58, No. 10, pp. 4526-4537, 2011. 


\section{Accepted Paper}

[33] E. Lopez, H. Opazo, L. Garcia, and P. Bastard, "Online reconfiguration considering variability demand: applications to real networks", IEEE Trans. Power Syst., vol. 19, no. 1, pp. 549-553, 2004.

[34] L. Kane, G. Ault, S. Gill, "An assessment of principles of access for wind generation curtailment in active network management schemes", in Proc. of 22nd International Conference on Electricity Distribution (CIRED), Stockholm, Sweden, June 2013.

[35] B.A. McCarl, "GAMS User Guide", Version 23.8, 2012. Available online: www.gams.com

Florin Capitanescu graduated in Electrical Power Engineering from the University "Politehnica" of Bucharest in 1997. He obtained the Ph.D. degree from the University of Liège in 2003. His main research interests include the application of optimization methods in the field of planning, operation, and control of transmission and distribution systems.

Luis F. Ochoa (S'01-M'07-SM'12) received the B.Eng. degree from UNI, Lima, Peru, in 2000 and the M.Sc. and Ph.D. degrees from UNESP, Ilha Solteira, Brazil, in 2003 and 2006, respectively. He is a Lecturer in Smart Distribution Networks at The University of Manchester, Manchester, U.K. His current research interests include network integration of distributed energy resources and future low-carbon distribution networks.

Harag Margossian received his Bachelor of Engineering and Master of Science degrees from the American University of Beirut (Lebanon) in 2008 and the Royal Institute of Technology (Sweden) in 2010 respectively. His main areas of interest include power system operation, protection and modelling. $\mathrm{He}$ is currently pursuing a joint $\mathrm{PhD}$ at the University of Luxembourg and K.U. Leuven.

Nikos D. Hatziargyriou is professor at the Power Division of the Electrical and Computer Engineering Department of the National Technical University of Athens. From February 2007 to September 2012, he was Deputy CEO of the Public Power Corporation (PPC) of Greece, responsible for Transmission and Distribution Networks, island DNO and the Center of Testing, Research and Prototyping. He is Fellow Member of IEEE, past Chair of the Power System Dynamic Performance Committee and Chair of CIGRE SC C6 "Distribution Systems and Distributed Generation". He is co-chair of the EU Advisory Council of the Technology Platform on SmartGrids. He is author of more than 160 journal publications and 500 conference proceedings papers. His research interests include Smartgrids, Microgrids, Distributed and Renewable Energy Sources and Power System Security. 